\title{
Voronoi Treemaps for the Visualization of Software Metrics
}

\author{
Michael Balzer \\ University of Konstanz, Germany University of Konstanz, Germany \\ Claus Lewerentz \\ Brandenburg University of Technology Cottbus, Germany
}

Abstract

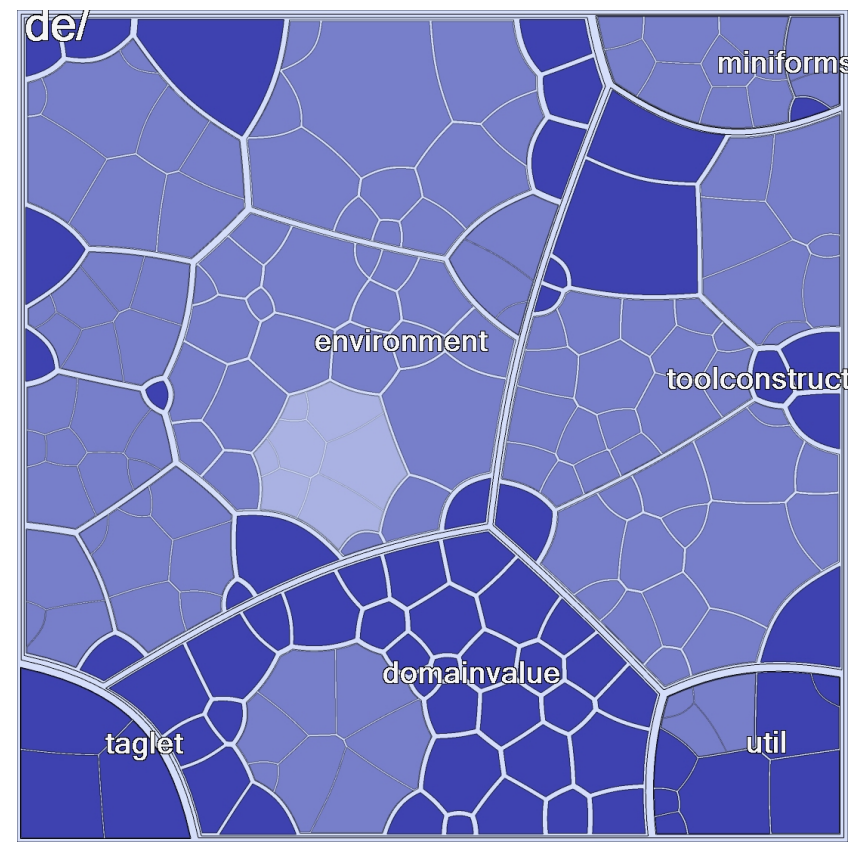

In this paper we present a hierarchy-based visualization approach for software metrics using Treemaps. Contrary to existing rectangle-based Treemap layout algorithms, we introduce layouts based on arbitrary polygons that are advantageous with respect to the aspect ratio between width and height of the objects and the identification of boundaries between and within the hierarchy levels in the Treemap. The layouts are computed by the iterative relaxation of Voronoi tessellations. Additionally, we describe techniques that allow the user to investigate software metric data of complex systems by utilizing transparencies in combination with interactive zooming.

CR Categories: D.2.8 [Software Engineering]: Metrics-; I.3.8 [Computer Graphics]: Applications-;

Keywords: Treemaps, Software Metrics, Voronoi Diagrams

Konstanzer Online-Publikations-System (KOPS)

URL: http://www.ub.uni-konstanz.de/kops/volltexte/2007/2417/ URN: http://nbn-resolving.de/urn:nbn:de:bsz:352-opus-24173

\section{Introduction}

Software systems are very complex hierarchical structures consisting of thousands of entities and millions of lines of code. Typical hierarchy levels of software entities are nested subsystems, packages, modules, functions, classes, methods, and attributes, whereby in large systems one may find up to 20 or more levels. In particular, object-oriented software systems are constructed using an explicit and rich hierarchical structure provided by modelling and programming languages like UML or Java/C++. Good quality assurance of these often extensive and complex systems, their components, and their development process, has become a decisive competitive advantage. The concerning methods and tools that support the developers in the stages of analysis, design, implementation, and documentation of the software, are therefore of fundamental importance.

Software metrics are quantitative measures of the degree to which a software system, a component, or process possesses a given attribute [of the IEEE Computer Society 1990]. They allow the identification of problem areas, the illustration of tendencies, and thereby help to improve the quality of software products as well as to increase the efficiency of the development process. In the context of program comprehension and quality analysis of large software systems, a enormous number of software metrics have been defined. These are used to quantitatively characterize properties of software entities, and to provide numerical abstractions of the entities themselves [Pfleeger et al. 1997]. Examples of software metrics are the widely used lines of code measure or the number of public attributes in a class.

In the field of software visualization different approaches exist to address the problem of creating visual representations of hierarchical software systems. 2D and 3D graphs focus on the relational structure of software entities including the hierarchy aspects [Lewerentz and Noack 2003; Noack 2003]. In such approaches metrics values are represented by visual attributes like size or color of graph nodes denoting the software entities. Other tools like CodeCrawler [Demeyer et al. 1999] focus on the metrics aspect, especially to represent multiple metric values for one software entity. A well known early tool for visualizing software metric data is SeeSoft [Eick et al. 1992], which arranges lines of code as pixels in a box, whereby their color stands for a certain value of a chosen metric. The newer tool Visual Insight [Eick et al. 2002] offers some visualizations for software metrics, although these representations are mostly bar, line, and pie charts, matrix views, and graph representations. However, the hierarchy of the entities as a fundamental characteristic of software systems is ignored by these and many other existing visualization tools. Most of the metrics-based approaches do neither support the hierarchical structure nor the requirements to create metrics-based views on higher abstraction levels. Additionally, an important advantage of using the hierarchy in the visualization is to clearly represent even extensive software systems [Balzer et al. 2004].

One of the rare tools in the domain of software visualization that follows this hierarchy-based approach is GAMMATELLA [Orso et al. 2003]. It visualizes program-execution data for deployed software by using Treemaps [Johnson and Shneiderman 1991], an in 
the information visualization community well-known and popular approach for hierarchical data. Here a given area is recursively subdivided by a given hierarchy without producing holes or overlappings. The existing layout algorithms for Treemaps are solely restricted to rectangular subdivisions, which however are disadvantageous with respect to the aspect ratio between width and height of the objects and the identification of boundaries between and within the hierarchy levels.

In this paper we present a method allowing us to generate Treemaps consisting of arbitrary polygons that enable a meaningful visualization of software metrics based on the hierarchy of a software system. In the following section we address the data and the characteristics of the visualized software metrics. In Section 3 previous work on Treemaps is outlined. Our approach of Voronoi Treemaps is presented in Section 4. Section 5 deals with assisting the user in the exploration of the data in the Treemaps. Section 6 summarizes our approach and discusses our future work. Examples of our Voronoi Treemap visualizations of software metrics are shown in Section 7.

\section{Underlying Data}

Our structural models of object-oriented software distinguish five types of software entities: packages, classes, methods, attributes, and files. Each package can contain other packages, classes, and files. Each class can contain other classes, methods, and attributes. The schema of the models is shown in Figure 1.

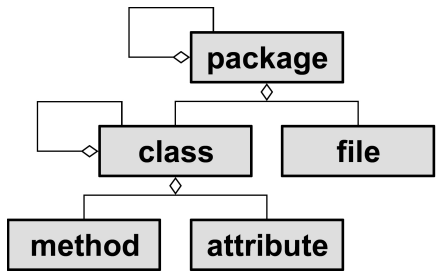

Figure 1: Schema for hierarchical models of object-oriented software systems

Such models can be automatically extracted from the source code of object-oriented software systems. In our experiments we used the tool Sotograph [Software-Tomography $\mathrm{GmbH}$ ] to extract the models from Java programs. The extracted models are stored in files in Rigi Standard Format [Wong 1998]. Through the separation of extraction from visualization, and the use of a standard exchange format, it is possible to visualize data from many sources. In particular, they can be applied to software in any programming language for which an appropriate extractor is available.

After extracting the models we possess a number of different software metrics for the different entity types. They range from the indication of size of the entities over the use by other entities, to cyclic dependencies, and many more. All of them are quantitative measures, which means that they can be counted, compared, ordered, and summated. To represent these software metrics with Treemaps, they are aggregated upward in the hierarchy. For example, a class consists of several methods. All methods have a given metric value that illustrates the number of calls of this method, and the sum of calls of all contained methods is assigned to the class. Likewise these metric values are propagated to the package level. Thereby the user is able to identify the packages and classes with frequently used methods immediately on a package or class level without having to examine the methods directly.

\section{Previous Work on Treemaps}

Treemaps were introduced by Shneiderman and Johnson in 1991 [Johnson and Shneiderman 1991]. Originally designed to visualize files on a hard drive, Treemaps have been applied to a wide variety of domains ranging from financial analysis [Jungmeister and Turo 1992; Wattenberg 1998] to sports reporting [Jin and Banks 1997]. The basic idea is to subdivide a given area without producing holes or overlappings. Therefore the area is alternately divided horizontally and vertically according to the hierarchy of the objects and the given proportion between the considered objects. Figure 2 illustrates this method with a simple example. Each node has a name and an associated size. The Treemap is constructed via recursive subdivision of the initial rectangle. The size of each sub-rectangle corresponds to the size of the node. The direction of the subdivision alternates per level: first horizontally, next vertically, again horizontally, etc. The initial rectangle is partitioned into smaller rectangles, so that the size of each rectangle reflects the size of the leaf. As a result of its construction, the Treemap reflects the structure of the tree. This original Treemap layout algorithm is called Slice-and-Dice.

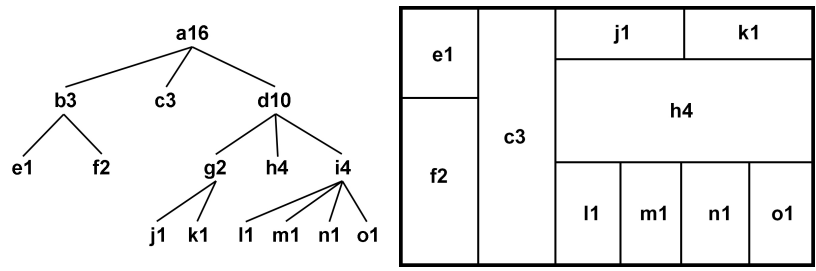

Figure 2: Tree diagram and corresponding Treemap

A negative effect in these layouts is however, that the subdivision in each step is solely done in one dimension. As result, thin elongated rectangles with a high aspect ratio between width and height emerge, if many objects or objects with high diversity in size are considered. Such long rectangles are difficult to see, select, compare in size, and label [Turo and Johnson 1992; Bruls et al. 2000]. Figure 3 presents an example.

This issue is addressed by Ordered Treemaps [Shneiderman and Wattenberg 2001], Squarified Treemaps [Bruls et al. 2000], Clustered Treemaps [Wattenberg 1999], and some other layout algorithms. These layouts subdivide the area of the initial rectangle in one step by both dimensions, meaning horizontally and vertically at the same time. The main optimization criterion of these layout algorithms is the approximation of the sub-rectangles to the shape of a square, whereby the aspect ratio between width and height of each rectangle converges to one. Aside from the aspect ratio criterion, other criteria are considered as well. For example the order of the objects or the nearness to a given point in the initial area, whereby the other criteria are often closely related to the respective application domain. A demonstrative member of this group of advanced Treemap layout algorithms is presented in Figure 4, showing the same data set as in Figure 3 with a Squarified Treemap layout that is currently the favored layout algorithm for Treemaps.

Another problem in this group of algorithms is illustrated in Figure 4: it is hard to differentiate if two neighbor objects are siblings or far away in the hierarchy. The problem is provoked by the square-like shape of the rectangles, and because the edges are only horizontally and vertically aligned, whereby the edges of the different objects appear to run into each other. This effect may be reduced, but can not be prevented by using borders and/or Cushion Treemaps [van Wijk and van de Wetering 1999]. A solution for this problem is the layout of Treemaps based on non-rectangular 


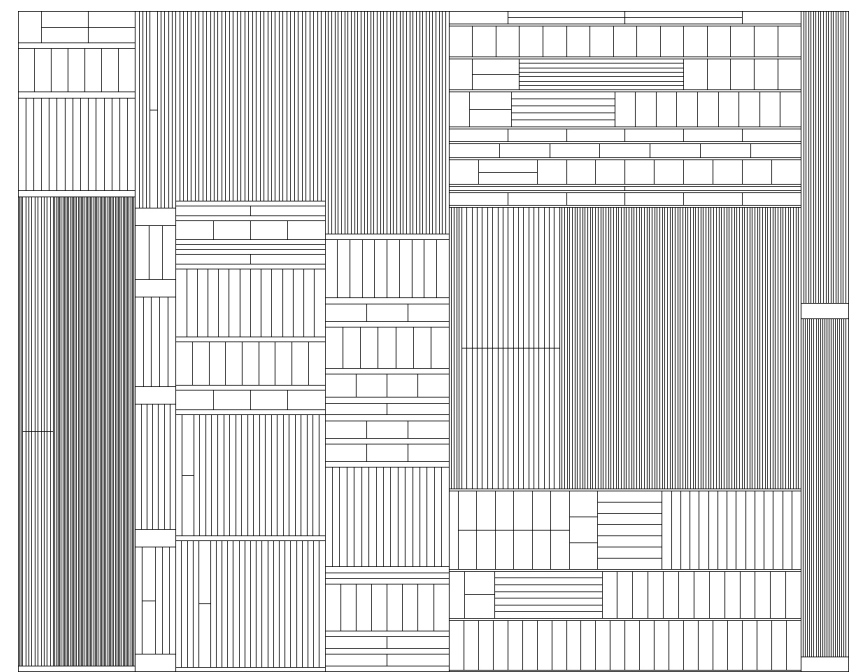

Figure 3: Aspect ratio problem of the original Slice-and-Dice Treemaps

objects. At this time such an approach does not exist, except in ETMaps [Roussinov and Chen 1998] where only composite shapes of rectangles are assembled. So far all Treemap layouts are restricted to axis-aligned rectangular shapes. Hence, we present our approach for polygon-based Treemaps in the next section, in which Treemaps with non-regulars shapes are generated.

\section{Voronoi Treemaps}

What are the constraints and optimization criteria for the shape of Treemap objects? Firstly, the distribution of the objects must fully utilize the given area, by avoiding holes and overlappings. Secondly, the objects should distinguish themselves, meaning they should have irregular shapes and the edges of the different objects should not run into each other. Thirdly, the objects should be compact, which means that the aspect ratio between their width and height should converge to one.

Obviously polygons can be used. A polygon is defined as a closed plane figure with $n$ sides. Special cases of polygons are triangles with $n=3$, rectangles, squares, or quadrilaterals with $n=4$, pentagons with $n=5$, and so on. Every polygon can be divided into smaller polygons, hereby satisfying the first constraint. Polygons can have arbitrary shapes and polygons with many edges can approximate curves, which refers to the second and third optimization criterion.

The principle structure of our layout algorithm is similar to the original Treemap layout algorithm. The first step is to consider the objects of the top level in the hierarchy. These objects are distributed in the given area - mostly a rectangle, but other shapes are possible, too. The output is a set of polygons. For the next hierarchy level, this algorithm is performed recursively within the according polygons of the considered objects in the hierarchy, and so on.

We now need a layout method, that allows us to divide a given area into polygons under consideration of the given optimization criteria. The basic idea is to use Voronoi tessellations. With their help, we are able to perform an iterative relaxation of a specified number of objects with corresponding sizes in a given polygonal area. This method and the underlying theory of Voronoi tessellations is

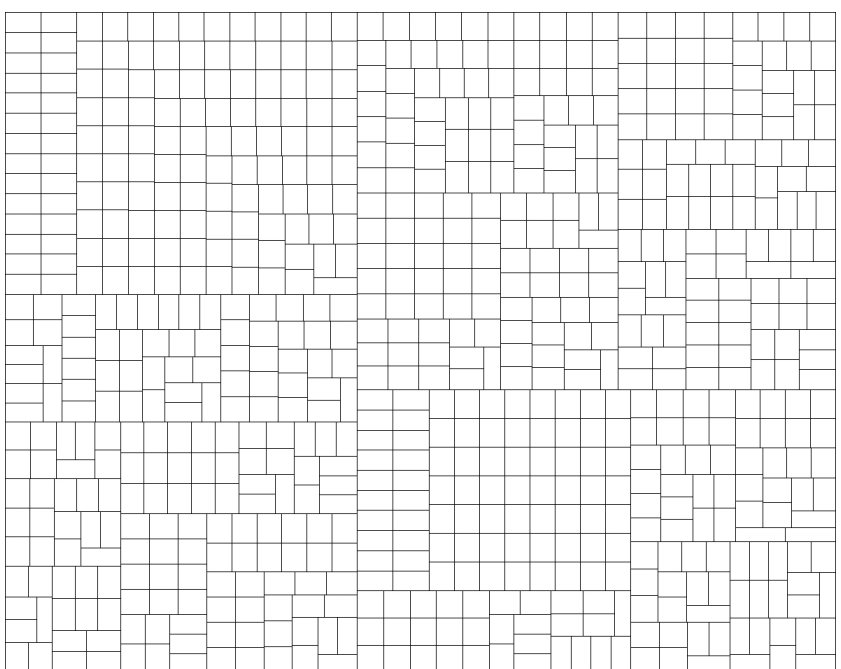

Figure 4: Hierarchy level seperation problem of Squarified Treemaps

explained in Section 4.1. However, here the problem is that for the advanced Voronoi metric used by us, it is non-trivial to compute the Voronoi tessellation analytically. For our application it is quiet sufficient to compute an approximation on a numerical basis. As a consequence thereof we must extract polygons out of this numerical computed Voronoi tessellations. This step is explained in Section 4.2. We need these polygons especially to perform the layout in the next lower hierarchy level recursively and to display the Treemap in general.

\subsection{Centroidal Voronoi Tessellation}

Let $P:=\left\{p_{1}, \ldots, p_{n}\right\}$ be a set of $n$ distinct points, where $2<n<\infty$, in $\mathbb{R}^{2}$ with the coordinates $\left(x_{p_{1}}, y_{p_{1}}\right), \ldots,\left(x_{p_{n}}, y_{p_{n}}\right)$. These points are the Voronoi sites. According to [de Berg et al. 2000; Okabe et al. 1992], we define the Voronoi tessellation of $P$ as the subdivision of $\mathbb{R}^{2}$ into $n$ cells, one for each site in $P$, with the property that a point $q$ lies in the cell corresponding to a site $p_{i}$ if and only if distance $\left(p_{i}, q\right)<$ distance $\left(p_{j}, q\right)$ for each $p_{i}, p_{j} \in P$ with $i \neq j$. The denotation distance $(p, q)$ represents a specified distance function between the two points $p$ and $q$ - mostly the Euclidian metric is used, which is defined as

$$
\operatorname{distance}(p, q):=\sqrt{\left(x_{p}-x_{q}\right)^{2}+\left(y_{p}-y_{q}\right)^{2}},
$$

but others such as the Manhattan metric or the Maximum metric, are possible as well. All points of a cell form a Voronoi polygon.

A Centroidal Voronoi Tessellation (CVT) [Du et al. 1999] is a Voronoi tessellation of a given set, whereby the associated generating points are centroids (centers of mass) of the corresponding Voronoi polygons. These tessellations represent arrangements similar to Poisson disc distributions. The CVT is computed by determining the Voronoi tessellation of a given point set, then each point is moved into the mass center of its assigned Voronoi polygon, and these two steps are repeated iteratively until the error between all point positions and the mass centers of their Voronoi polygons is below a given $\varepsilon$. Figure 5 shows an example of a Voronoi tessellation of 20 random points on the left, and the associated CVT on the right - traces illustrate the movements of the points during the computation of the CVT. 

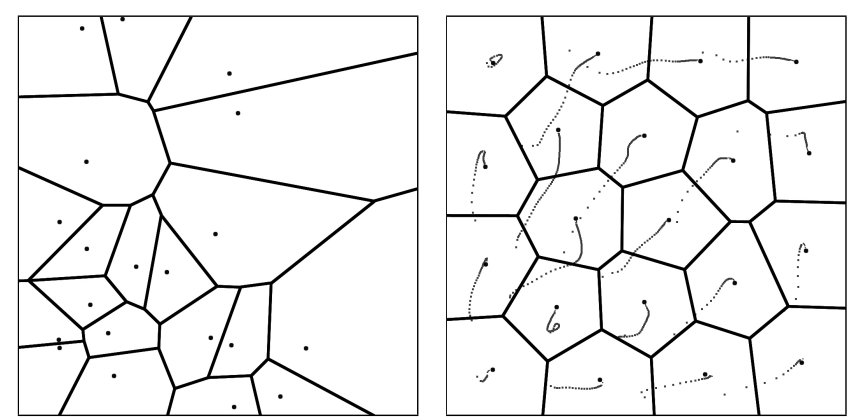

Figure 5: Voronoi tessellation of 20 random points and the associated CVT (traces illustrate the point movements during the computation of the CVT)

With this basic CVT we are able to compute Treemap layouts in which every leaf node in the hierarchy has the same value. However, it is our goal to generate layouts in which the objects can have different values. Hence we need to extend the distance metric by adding a size parameter to every point. This corresponds to the computation of Voronoi tessellations with circles as generator objects, where the size parameter is the radius. The resulting distance measure function is defined between a point $q$ and a circle $c$ with the center $p$ and the radius $r$ as

$$
\operatorname{distance}(c, q)_{\text {Circle }}:=\operatorname{distance}(p, q)-r \text {. }
$$

Additionally, the radii of the circles are not specified by precise values, but rather exist only as relations between all considered circles. Their absolute radii result adaptively from the momentary positions of the circles. This step is necessary because overlapping circles would otherwise generate uncontinuous Voronoi polygons. Therefore a maximum factor $m$ is determined, so that no two circles $c_{1}\left(p_{1}, r_{1}\right)$ and $c_{2}\left(p_{2}, r_{2}\right)$ exist with

$$
\text { distance }\left(p_{1}, p_{2}\right)-\left(r_{1}+r_{2}\right) * m<0 \text {. }
$$

Factor $m$ is then multiplied with the relative radius of each circle, the result being the absolute radius. By creating CVTs fulfilling these constraints, distributions of circles are developed under the criterion of 'Maximum Growth'.

With this modified CVT we have not yet reached our goal, because the values of the objects only correspond to the radii of the generator circles and not to the sizes of the surface areas of the dedicated Voronoi polygons. However, the key to success is to use the radii to control the area sizes of the Voronoi polygons during the iterative computation of the CVT. Analog to the modification of the position in the classic CVT computation, we additionally change the radius of every generator circle according to the relative size of the Voronoi polygon to the total area size. For example, if the value of an object in our hierarchy is $20 \%$ of the sum of all object values in the current hierarchy level, but in the last iteration step the dedicated Voronoi polygon covers only $16 \%$ of the overall area, we will try to increase the area size of the Voronoi polygon in the next iteration from $16 \%$ to $20 \%$ by increasing the radius of the generator circle by $25 \%$. Due to the facts that the relation between the radius of a circle and the area size of the dedicated Voronoi polygon is not a linear dependency, and the radii and positions of the other generator circles are changing at the same time, we will most likely not achieve the correct area size in the next step. However, we may presume that a better approximation is obtained. By performing these adjustments of the radii in every iteration step, the computation will end up in a stable state, whereby the error between the designated relative value of every object in the hierarchy and the relative area size of the dedicated Voronoi polygon is below a given $\varepsilon$.
To further clarify the above procedure it should be noted, that firstly, the radius of a circle may have values below zero - formally this object is not a circle, but in our application this abstraction is useful and even essential. Secondly, beside the mentioned simple adjustment of the radius according to the area size error, it is necessary to observe certain cases where the radius is or is nearby zero, and where the radius needs to be switched from positive to negative, or vice versa, to obtain better approximations.

Figure 6 illustrates this iterative procedure with six objects, each having a different value. After 104 iterations the Voronoi polygon area size error $\delta_{A}$ for every object was less than 0.01 . At iteration 217 a stable state with a Voronoi polygon area size error $\delta_{A}<0.001$ for every object was reached. During the computation the circles have altered their sizes, relating to the changes of the positions and radii of the other circles. The changing of the maximum area size error of an object and the overall area size error is illustrated in Figure 7. The computation time was less than one second on a Pentium 4.

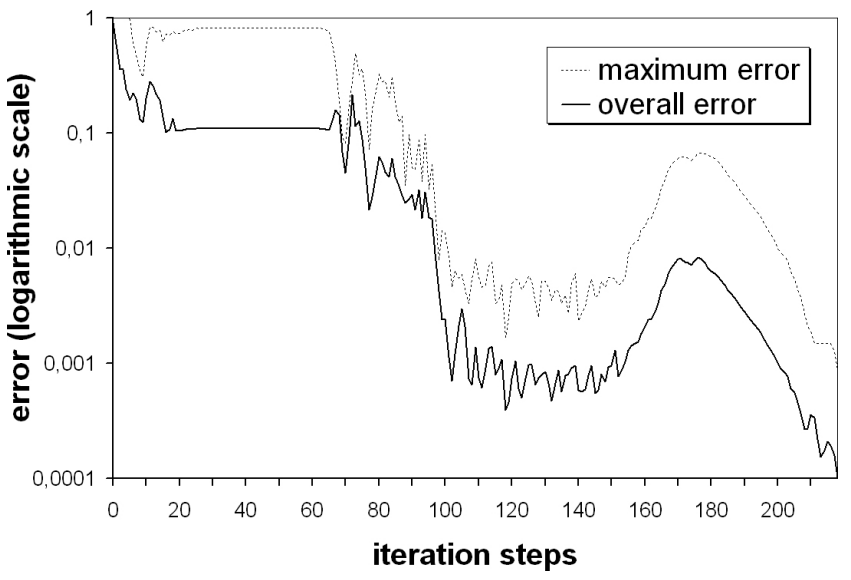

Figure 7: Convergence of the maximum area size error of an object and the overall area size error during the computation of the CVT in Figure 6. At a maximum error of $\delta_{A}<0.001$ the computation stopped.

As already mentioned in Section 4, it is non-trivial to compute this Voronoi tessellations analytically, instead we calculate it numerically. Hence, we use a set of sample points that is a CVT itself in compliance to the sampling theorem this produces much better results than a regular grid or random points [Okabe et al. 1992]. The size of this set has to be appropriate for the number of generator objects - in our experiments we used sets with sizes between $10^{\prime} 000$ and 100'000 points. Before we start the computation of the CVT, we first clip the set at the outer polygon that describes the total available area for the current layout step. Following [Hoff et al. 1999], in every iteration step for every sample we determine the nearest circle according to the distance $(c, q)_{\text {Circle }}$ metric. Then, for every circle we average the positions of the samples for which the considered circle is the nearest to obtain the mass center of the according Voronoi polygon. With this method we obtain good approximations for Voronoi tessellations which satisfy the needs for computing the CVT. Though this method is not precise enough to extract the correct polygonal representations of the Voronoi cells. Therefore we must use the method presented in the next section. 

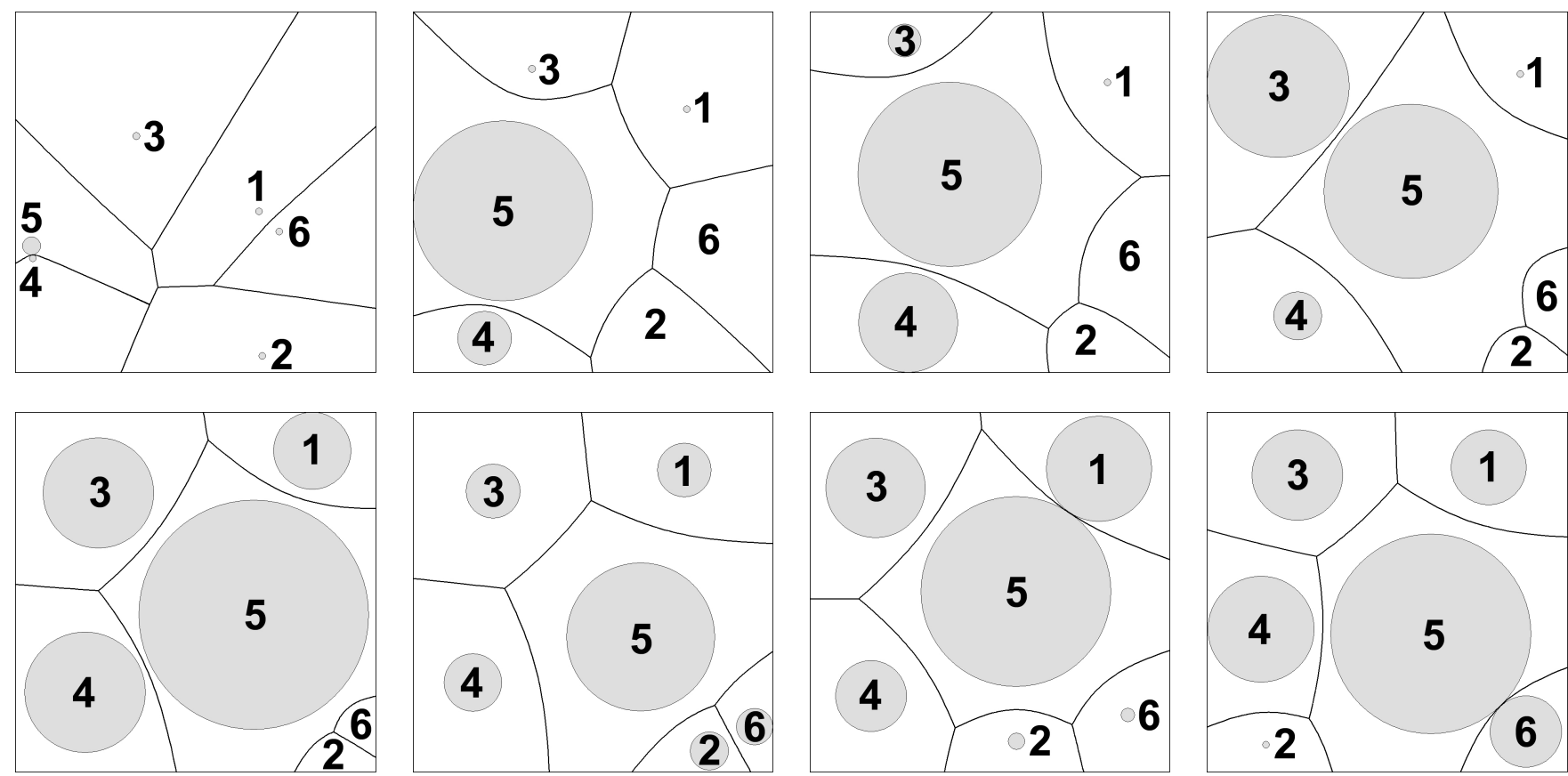

Figure 6: Iterative computation of a CVT of six objects with the Euclidian distance metric based on circles. A stable state with a Voronoi polygon area size error $\delta_{A}<0.001$ was reached in 217 steps. Iteration steps from top left to bottom right: 0 (start), 2, 6, 10, 14, 67, 76, 217 (end).

\subsection{Polygon Extraction}

After the CVT has been computed, we have to extract the Voronoi polygons. Contrary to Section 4.1 we do not want to use a set of sample points, because a polygon extraction on this basis produces zigzag lines. Instead we will construct the curve segments between the Voronoi cells directly. A curve segment $e$ between two Voronoi cells, specified by the two circles $c_{1}$ and $c_{2}$, is defined as a set of points $P$ in $\mathbb{R}^{2}$ where every $p \in P$ fulfills the following two constraints:

$$
\operatorname{distance}\left(p, c_{1}\right)_{\text {Circle }}=\operatorname{distance}\left(p, c_{2}\right)_{\text {Circle }}
$$

distance $\left(p, c_{1}\right)_{\text {Circle }}<\operatorname{distance}\left(p, c_{i}\right)_{\text {Circle }}$ for $i \notin\{1,2\}$.

Obviously a curve segment between circles of the same radius is a straight line, and between circles of different radius a parabola. To construct these curve segments we determine all curves between any two circles, select the relevant curves, clip them against each other and the outer polygon, and finally merge them to polygons.

Since a polygon consists of a limited number of straight line edges, it is adequate to choose a limited number of points $p_{i} \in P$ as well. Given two circles $c_{\text {small }}$ and $c_{\text {large }}$, a reasonable criterion is to sample the curve by the angle $\alpha$ and the circle $c_{\text {small }}$ with $r_{\text {small }} \leq r_{\text {large }}$. This means to shoot $n$ rays from $p_{\text {small }}$, where the angle between the rays $t_{i}$ and $t_{i+1}$ is an adequate $\alpha$, whereas $t_{n+1}=t_{1}$. This procedure enables a curvature-adaptive sampling. On every ray $t$ we calculate a point $p$ which satisfies the first constraint for the curve segment $e\left(c_{\text {small }}, c_{\text {large }}\right)$, with the side constraint that for every $p_{i} \in P$ distance $\left(p_{i}, c_{\text {small }}\right)_{\text {Circle }}>$ distance $\left(p, c_{\text {small }}\right)_{\text {Circle }}$ with $p \neq p_{i}$, and distance $\left(p, c_{\text {small }}\right)_{\text {Circle }}<\infty$. The connection of these points according to the sampling order results in the designated curve, which defines the border between the Voronoi cells of $c_{\text {small }}$ and $c_{\text {large }}$. The left image in Figure 8 shows these curves for every pair of circles.
After approximating the curves between all pairs of circles in the manner described, we have to select the relevant curves. On a relevant curve $e\left(c_{1}, c_{2}\right)$ exists a point $p$ with the property that for every circle $c_{i}$ by $i \notin\{1,2\}$ distance $\left(p, c_{i}\right)_{\text {Circle }}>$ distance $\left(p, c_{1}\right)_{\text {Circle }}$. Non-relevant curves cannot contain curve segments for the Voronoi tessellation due to the definitions in Section 4.1. The state after discarding the non-relevant curves is illustrated in the center image in Figure 8 .
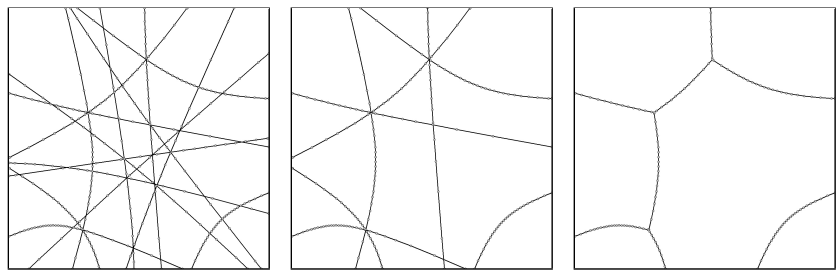

Figure 8: Extraction of Voronoi polygons: determining all curves (left), discarding non-relevant curves (center), clipping (right)

The determined relevant curves are then clipped at the outer polygon by starting from a point at the curve within the polygon, and successively intersecting all edges of the curve with all edges of the outer polygon in both directions of the starting point. If an intersection is found on one side, all points beyond this intersection are discarded, the intersection point is added to the curve, and the search for intersections at the considered side of the polygon is stopped. Now we have a set of relevant curve segments which are completely within the outer polygon. In the next step the segments are clipped taking the other segments into consideration. Following the above constraint while looking for relevant curves, for every segment we search one point whose two nearest neighbors are those circles which have created this segment. Starting from this point, we clip every edge of the segment $e_{1}\left(c_{1}, c_{2}\right)$ at all edges of the other segments $e_{i}\left(c_{j}, c_{k}\right)$ at both sides of the starting point, whereby 
$i \neq 1$, and $j \in\{1,2\}$ or $k \in\{1,2\}$. If an intersection is found on one side, again all further points are discarded, the intersection point is added, and the search at this side is stopped. The right image in Figure 8 shows the segments after the clipping process.

Now we can assemble the final Voronoi polygons by combining the remaining segments and the segments of the outer polygon. Instead of looking for possible connection points, we utilize the fact that as a result of the defined distance function each Voronoi polygon has the following characteristic: the straight line edge between the center of the circle that is associated to the Voronoi polygon and every point of this polygon does not intersect any of the polygon edges. Thus, we first calculate for each point $p$ of the outer polygon the circle $c$ for which distance $(p, c)_{\text {Circle }}<$ distance $\left(p, c_{i}\right)_{\text {Circle }}$ for $c \neq c_{i}$, and add this point to the correspondent Voronoi polygon of circle $c$. Then we sort all points of the segments and of the outer polygon that are assigned to the respective circle by the angle of the straight line edge between the center of the circle and the considered point. Again it should be noted, that as a result of the curve sampling step, the described polygon extraction method is an approximation as well.

As the result we now have Voronoi polygons without holes and overlappings within a given outer polygon, whereby the area size of each polygon corresponds to the value of the assigned generator object in the hierarchy. Furthermore, these polygons are clearly distinguishable because of their irregular shapes, and their aspect ratio converges to one. This fulfills the constraints and optimization criteria of Treemaps, given in Section 4. Figure 9 presents the final Voronoi Treemap layout computed with our approach.

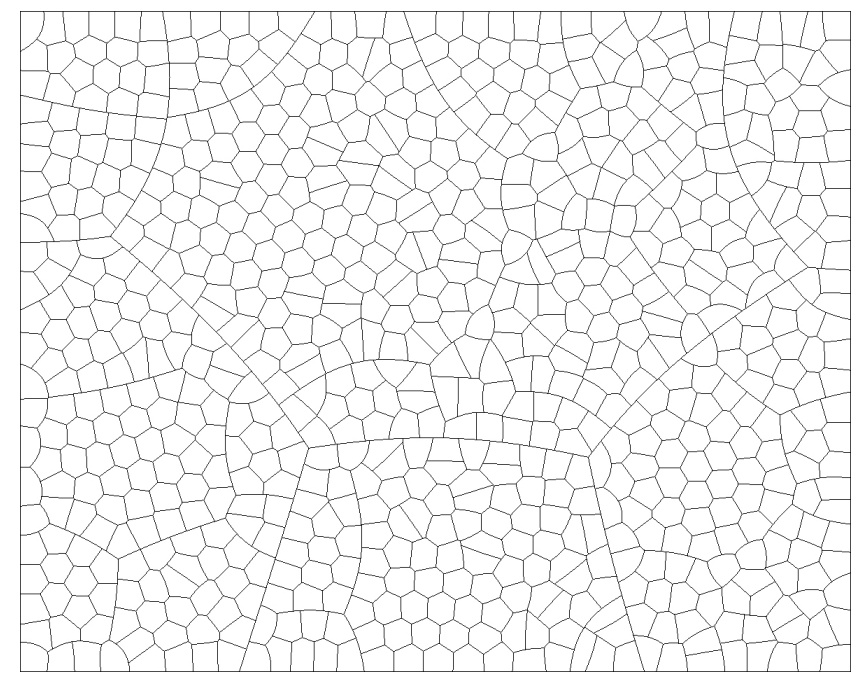

Figure 9: Final Voronoi Treemap layout with the same dataset as in Figure 3 and 4

\section{User interaction}

Als already mentioned, software systems can be very complex. Thus the Treemap layouts which represent the software metric data of these systems will also be very complex. For this reason, we developed and implemented two techniques for our software metric data visualization tool. The first technique allows the user to interact with the visualization. The second hides parts of the visualized data so not to overwhelm the user with the mass of information. The combination of these two techniques enables the effective ex- amination of large and complex data sets with thousands or even millions of entities.

Interactive Zooming: With our tool the user is able to explore the data analog to text based hierarchy browsers. At the beginning, the complete Treemap is presented, starting at the top hierarchy level. If the user is interested in a special part of the data he simply clicks in this area, whereby the object in the next lower hierarchy level relating to this area is selected. Following this selected object is shown in full size on the screen. Again the user can select a subarea for further investigation downwards the hierarchy, or he can move back upwards the hierarchy. For not losing the context in the visualization, the transition between two states is animated. Figure 10 illustrates this interaction scheme - borders have been added to every Voronoi polygon for a better differentiation between the objects.

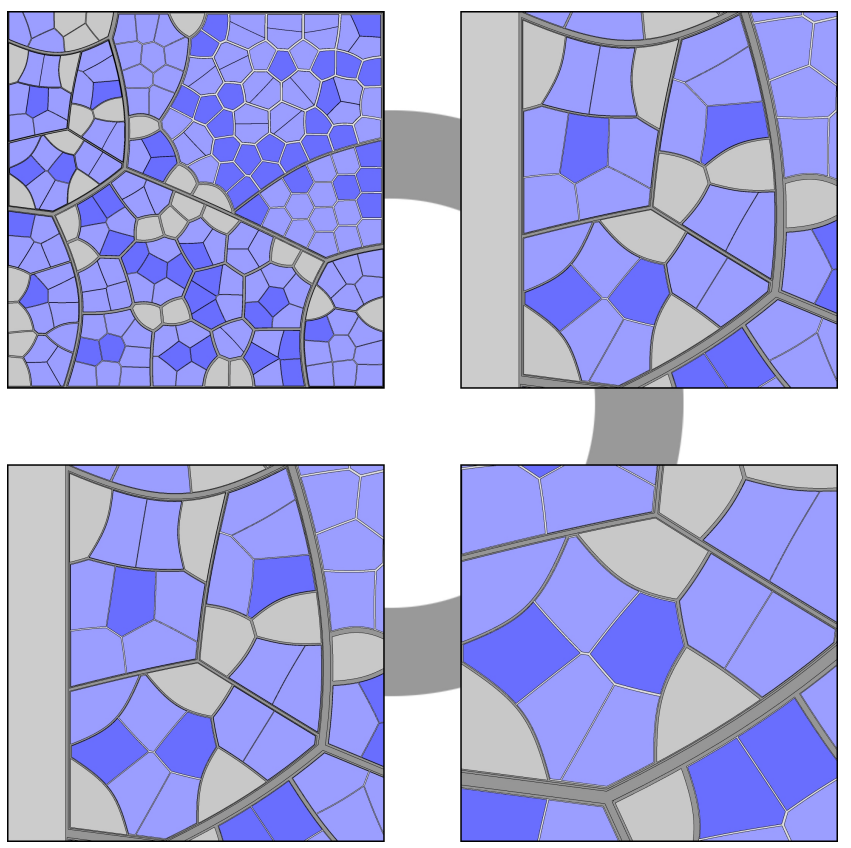

Figure 10: Zooming downwards and upwards the hierarchy

Transparencies for Level-of-Detail: Using a hierarchy for the software entities not only allows for better organizing them, but the hierarchy is also designed to give an abstract view to components of the software system. For that reason, it is not always desired to inspect the system down to the last attribute, but rather it is necessary to have these levels of abstraction in the visualization as well. To realize this abstracted views we utilize transparencies similar to [Balzer et al. 2004] in the following way: all objects at or above the current hierarchy level are fully opaque. For every step down the hierarchy, the transparency of the according objects is increased by a given value $\delta_{t}$. If this value is equal to or below zero, all objects on this level and all subordinate objects are not drawn. In the rendering step after assigning the transparency values, all objects are rendered in descending order according to the hierarchy, meaning that the objects of the first level are rendered at first, then the objects of the second level, and so on. The visual effect here is that the objects further down the hierarchy seem to disappear and objects which are $1 / \delta_{t}$ or more levels down the hierarchy are hidden. The change of the transparencies is animated when browsing through the hierarchy as well, whereby objects not suddenly pop up or disappear from one frame to another. In Figure 11 a data set with different $\delta_{t}$ is shown to demonstrate this information-based Levelof-Detail technique. 

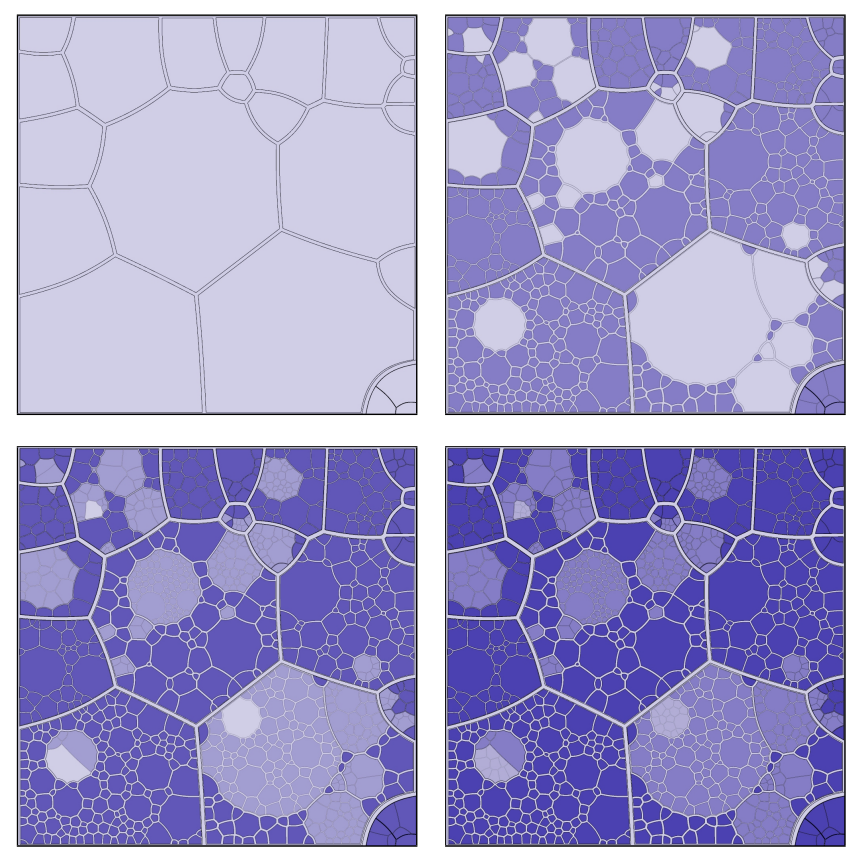

Figure 11: Using Transparencies for information-based Level-ofDetail

Beside these two techniques we implemented an adaptive and selective labeling and highlighting of the Treemap objects, which additionally supports the user by finding the information he is looking for. Examples are shown in the figures of Section 7.

\section{Conclusions and Future Work}

In this paper we presented an approach for visualizing software metrics. We introduced a new method for computing layouts of Treemaps, which are based on polygons and explained these method in-depth. Additionally, we described techniques that allows the user to investigate software metric data of complex software systems. Aside the domain of software visualization this combination of algorithms and techniques can also be used for other attributed hierarchical data.

In an informal user study of students and colleagues, we observed that the hierarchy and size parameters of the objects were better recognized with our polygon-based Treemaps, than the established algorithms presented in Section 3. Our estimation with regard to the figure of the Treemap objects were confirmed, meaning that the arbitrary shape of the polygons allows a much better differentiation than rectangles.

In the current situation the computation for the Treemap layouts is based on sampling. In this regard the computation is relatively slow, which means that the time for generating layouts of complex data sets with many thousand entities requires minutes instead of seconds for the established layout algorithms. But in our opinion this time is justifiable in reference to the obtained results.

Our future work will address three domains: firstly, we want to adapt other metrics than the Euclidian distance measure to our algorithm. Secondly, we aim for improving the polygon extraction part by solving this problem analytically. Thirdly, as a final result we want to integrate this technique in a software analysis environment.

\section{Results}

Finally, in the Figures 12 and 13 we want to present some demonstrative results for software metric visualizations that are generated with our Voronoi Treemap method including some additional information about the represented data and the used metrics. A color version can be found at the additional color plate.

The colors of the Treemap objects in the Figures 12 and 13 represent the entity type of the according objects in the hierarchy of the software system. Packages are light blue, classes are red, files are yellow, methods are dark blue, and attributes are green. The color of every object in the visualization is altered depending on the transparency of the objects and the color of the occluded objects.

\section{References}

Balzer, M., Noack, A., Deussen, O., And Lewerentz, C. 2004. Software landscapes: Visualizing the structure of large software systems. In Joint Eurographics and IEEE TCVG Symposium on Visualization, Eurographics Association, 261-266.

Bruls, M., Huizing, K., AND VAN WijK, J. 2000. Squarified treemaps. In Joint Eurographics and IEEE TCVG Symposium on Visualization, IEEE Computer Society, 33-42.

de Berg, M., van Kreveld, M., Overmars, M., And SCHWARZKOPF, O. 2000. Computational Geometry: Algorithms and Applications, 2nd ed. Springer-Verlag, Berlin, Germany.

Demeyer, S., Ducasse, S., And Lanza, M. 1999. A hybrid reverse engineering approach combining metrics and program visualization. In Proceedings of the 6th Working Conference on Reverse Engineering, IEEE Computer Society, 175-186.

Du, Q., Faber, V., And Gunzburger, M. 1999. Centroidal voronoi tessellations: Applications and algorithms. SIAM Review 41, 4, 637-676.

EICK, S. G., Steffen, J. L., And JR., E. E. S. 1992. Seesoft - a tool for visualizing line oriented software statistics. IEEE Transactions on Software Engineering 18, 11, 957-968.

Eick, S. G., Graves, T. L., Karr, A. F., Mockus, A., And SCHUSTER, P. 2002. Visualizing software changes. IEEE Transactions on Software Engineering 28, 4, 396-412.

Hoff, K., Keyser, J., Lin, M., Manocha, D., And Culver, T. 1999. Fast computation of generalized voronoi diagrams using graphics hardware. In Proceedings of the 26th Annual Conference on Computer Graphics (SIGGRAPH), ACM Press, 277-286.

JIN, L., AND BANKS, D. C. 1997. Tennisviewer: A browser for competition trees. IEEE Computer Graphics and Applications $17,4,63-65$.

Johnson, B., And Shneiderman, B. 1991. Tree maps: A space-filling approach to the visualization of hierarchical information structures. In IEEE Visualization, IEEE Computer Society, 284-291.

Jungmeister, W.-A., And Turo, D. 1992. Adapting treemaps to stock portfolio visualization. Tech. Rep. UMCP-CSD CS-TR2996, University of Maryland, College Park, Maryland 20742, U.S.A. 

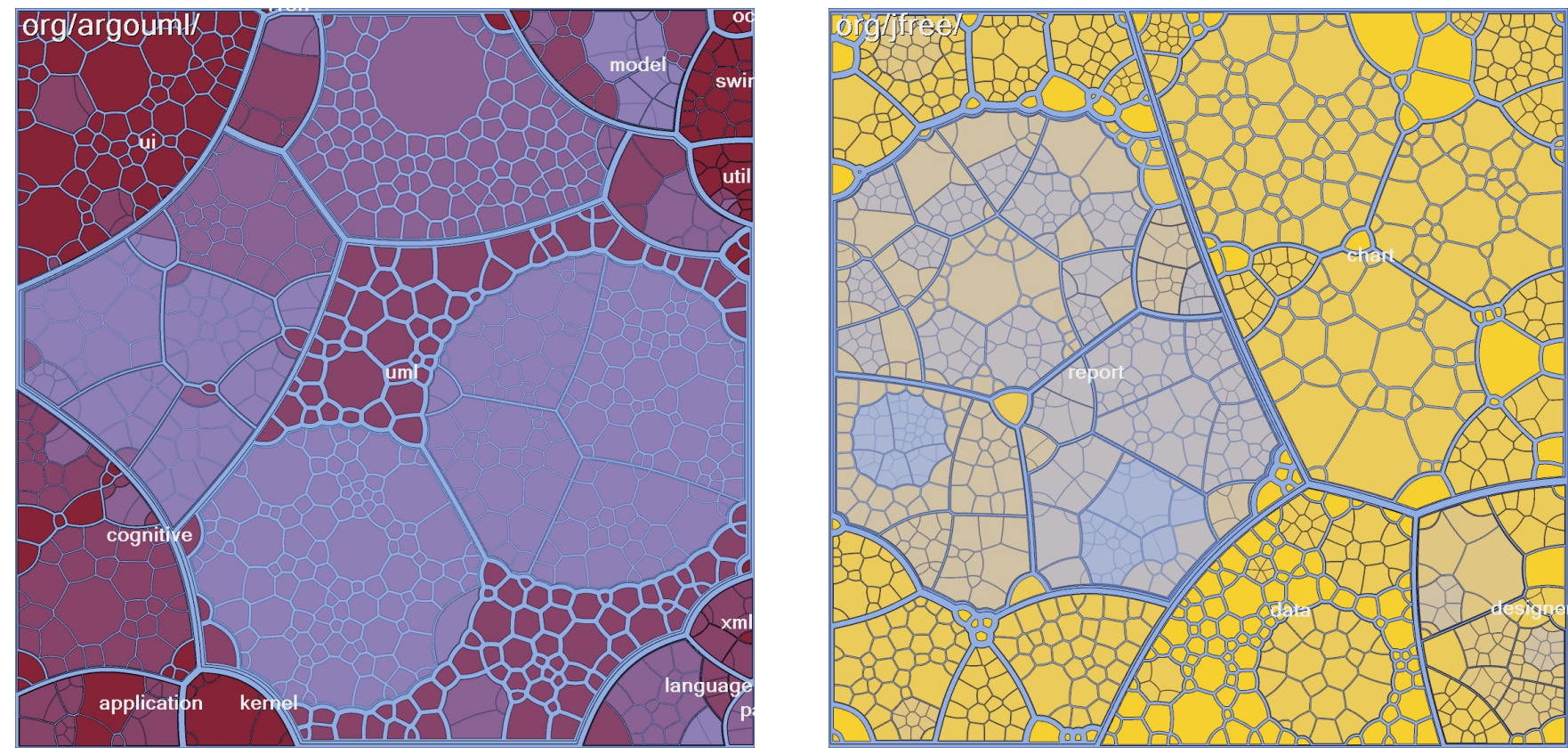

Figure 12: The left image visualizes the outbound calls of classes by other classes of the software system 'ArgoUML'. The right image visualizes the lines of code (LOC) of all files of the software system 'JFree'.

Lewerentz, C., AND NoACK, A. 2003. Crococosmos - 3D visualization of large object-oriented programs. In Graph Drawing Software, M. Jünger and P. Mutzel, Eds. Springer-Verlag, 279297.

NoACK, A. 2003. An energy model for visual graph clustering. In Proceedings of the 11th International Symposium on Graph Drawing, Springer-Verlag, 425-436.

OF THE IEEE COMPUTER SocIETy, S. C. C., 1990. IEEE standard glossary of software engineering terminology. IEEE Std 610.12-1990.

OKabe, A., Boots, B., And Sugihara, K. 1992. Spatial Tessellations: Concepts and Applications of Voronoi Diagrams, 2nd ed. John Wiley and Sons Ltd.

Orso, A., Jones, J. A., And Harrold, M. J. 2003. Visualization of program-execution data for deployed software. In Proceedings ACM 2003 Symposium on Software Visualization, ACM Press, 67-76.

Pfleeger, S. L., JefFery, R., Curtis, B., And Kitchenham, B. 1997. Status report on software measurement. IEEE Software $14,2,33-43$.

Roussinov, D., AND CHEN, H. 1998. A scalable self-organizing map algorithm for textual classification: A neural network approach to thesaurus generation. Communication and Cognition $15,1-2,81-112$.

Shneiderman, B., And Wattenberg, M. 2001. Ordered treemap layouts. In Proceedings of the IEEE Symposium on Information Visualization, IEEE Computer Society, 73-78.

SOFTWARE-TOMOGRAPHY http://www.softwaretomography.com.

TURO, D., AND JOHNSON, B. 1992. Improving the visualization of hierarchies with treemaps: Design issues and experimenta- tion. Tech. Rep. UMCP-CSD CS-TR-2901, University of Maryland, College Park, Maryland 20742, U.S.A.

VAN WiJK, J. J., AND VAN DE Wetering, H. 1999. Cushion treemaps: Visualization of hierarchical information. In Proceedings of the IEEE Symposium on Information Visualization, IEEE Computer Society, 73-78.

WATtENBERG, M., 1998. Map of the market, http://smartmoney.com/marketmap.

WATtEnBerg, M. 1999. Visualizing the stock market. In Extended Abstracts on Human Factors in Computing Systems, ACM Press, 188-189.

Wong, K. 1998. Rigi User's Manual, Version 5.4.4. http://ftp.rigi.csc.uvic.ca/pub/rigi/doc/. 

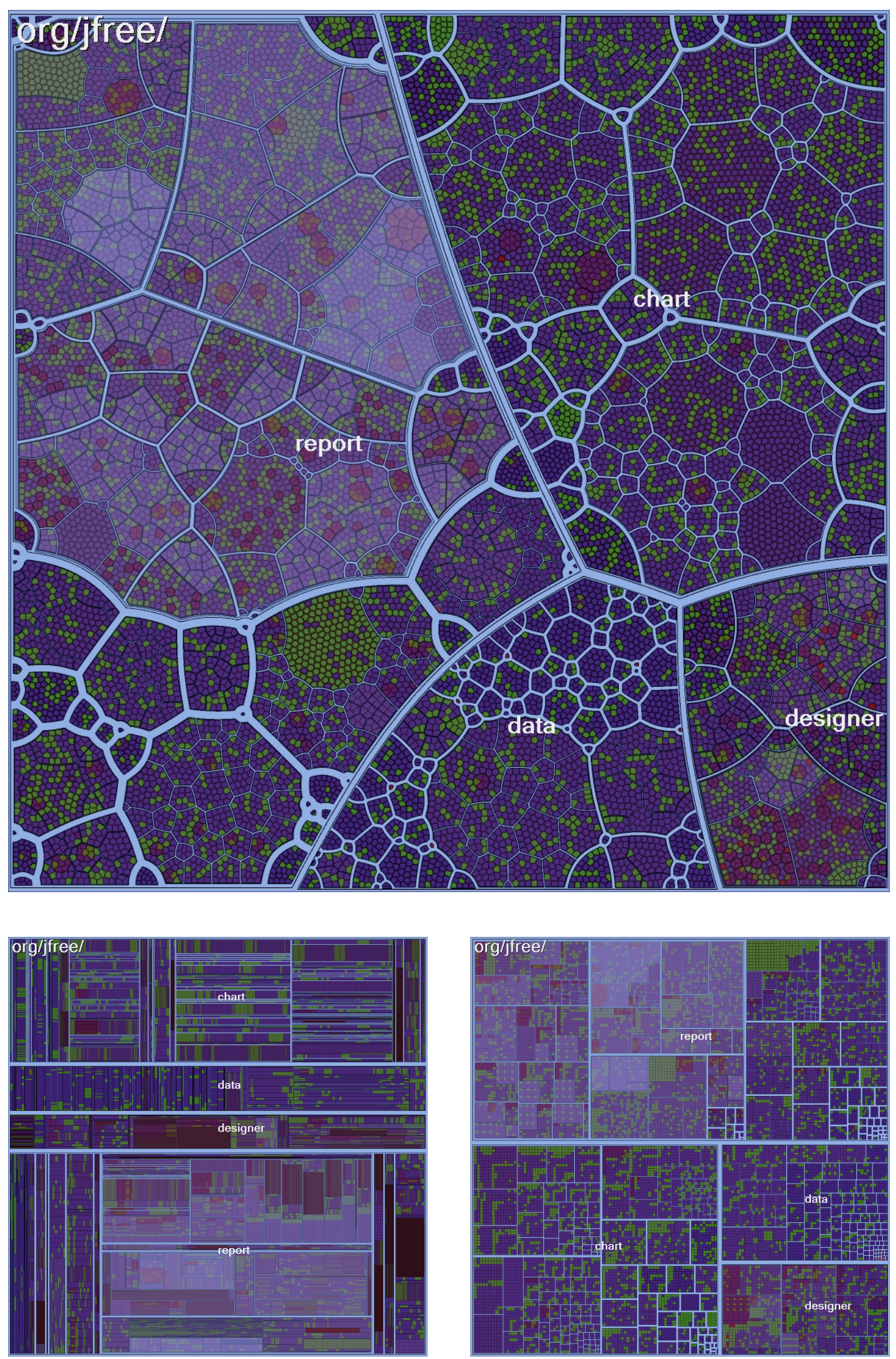

Figure 13: Visualization of the static structure of packages, classes, methods, and attributes of the software system 'JFree'. The top image uses the Treemap algorithm presented in this paper, the lower left uses the Slice-and-Dice algorithm, and the lower right is computed with the Squarified Treemap algorithm. 